\section{Intravascular leiomyoma misdiagnosed as deep vein thrombosis: A case report}

\author{
Yiheng Zhang, Feng Wen*, Zhen Liu and Zaiming Lu \\ Department of Radiology, Shengjing Hospital of China Medical University, No.36, Sanhao St., Shenyang, \\ 110000 China
}

\section{Abstract}

Background: Leiomyoma itself is not a rare disease, but it is rarely found intravascularly.

Case presentation: A 54-year old female sought medical help after noticing her leg being swelling. A diagnosis of deep vein thrombosis (DVT) was made and antithrombotic treatment was given after her initial imaging exam. Several days later, a contrast CT and sequential pathology revealed the real diagnosis was intravascular leiomyoma. The patient was discharged after a successful surgery.

Conclusion: Intravascular leiomyoma should not be confused with DVT.

\section{More Information}

*Address for Correspondence: Feng Wen, Department of Radiology, Shengjing Hospital of China Medical University, No.36, Sanhao St., Shenyang, 110000 China,

Tel: 008618940255717; Email: drwenf@126.com

Submitted: June 16, 2021

Approved: June 29, 2021

Published: June 30, 2021

How to cite this article: Zhang Y, Wen F, Liu Z, Lu Z. Intravascular leiomyoma misdiagnosed as deep vein thrombosis: A case report. J Radiol Oncol. 2021; 5: 042-045.

\section{DOI: 10.29328/journal.jro.1001037}

Copyright: () 2021 Zhang Y, et al. This is an open access article distributed under the Creative Commons Attribution License, which permits unrestricted use, distribution, and reproduction in any medium, provided the original work is properly cited.

Keywords: Case report; Vein Thrombosis; Leiomyoma; Diagnostic errors

\section{Check for updates}

OPEn ACCESS

\section{Background}

Intravascular leiomyoma is a rare condition that is reported exclusively in females [1]. The pathophysiology is not well established. The origin of this invasive benign tumor tends to be attributable to uterine tissue. According to a study by Li [2], only two percent of all patients did not have a normal uterus before their intravascular lesion was diagnosed.

Although intravascular leiomyomata are histologically benign, they may be found beyond the pelvic region. Therefore they have the potential to cause severe complications, such as obstruction of the heart outflow tract or vena cava, pulmonary embolism, even sudden death [3]. There are no specific symptoms of intravascular leiomyoma. Most of its clinical appearances are due to obstruction of the blood stream, such as lower extremities edema. For that reason, it and other intravascular tumors could be misdiagnosed as DVT, especially those tricky cases which happen in lower extremities vascular, such as the case we report here.

\section{Case presentation}

A 54-year-old female came to outpatient clinic for right lower extremity swelling after a 14 hours train trip one week before. The swelling was periodical, but it was deteriorating as the week passed by. A focused physical exam showed no significant changes of right lower muscle strength. When she sat, the swelling of her leg exacerbated dramatically, with lower extremity skin turning red. She also complained of occasional right leg weakness after squat position, which started six months before this visit. Her Wells' Score for DVT was deemed 3. The patient underwent surgery for osteomyelitis when she was 12 years old, which resulted in left leg atrophy. She had 2 offspring and denied any significant surgery or OB/GYN history. The patient was prescribed Rivaroxaban $20 \mathrm{mg}$ QD PO after her emergent lower extremity sonography suspected venous thrombus.

The patient was admitted in interventional ward when her symptoms had no change after one week's medication therapy. Though there were no abnormalities in her blood coagulation tests, she was given an inferior vena cava filter, and also a catheter was inserted inside the lesion. Several attempts at retrieving thrombi were made when the lesion was detected under DSA (Figure 1), but nothing was able to be dislodged. After her interventional procedure, Urokinase 0.3 million units Q12 was given through pedal vein, and Nadroparin 3800 unites was given subdermal. Another set of CBC and coagulation test were ordered 36 hours later; both came back normal. Her physical exams were roughly the same compared to admission.

The suspicion of misdiagnosis was raised, so a contrast CT of lower abdomen was complete two days later, one day after her Urokinase was discontinued. Her CT showed that a $30 \mathrm{x}$ $35 \times 45 \mathrm{~mm}$ oval-shape soft tissue nodule resided in the right iliac vein with local vein dilation. The lesion has an average 
CT value of $40 \mathrm{HU}$ ( 15 to $65 \mathrm{HU}$ ) on plain film, while enhanced heterogeneous in arterial phase (Figure 2). The patient was given Rivaroxaban $\mathrm{PO}$ for another two days before transfer to surgery unit. The surgery removed two pieces of light yellow tissue and one brownish tissue. The pathology (Figure 3) confirmed atypical leiomyoma (ER +, PR -, Desmin +, SMA + , Ki-67 10\%). Two days after surgery, the patient started to have mild right lower extremity swelling, which led to another sequence of Rivaroxaban 10mg PO BID before her follow-up ultrasound detected right iliac vein, right femoral vein and saphenous vein thrombus (Figure 4). This finding led to another seven days of Urokinase 0.2 million unites Q12 through intravenous catheter.

The patient was discharged two weeks after proper treatment and disappearance of her symptoms. She then was followed through telephone for the next three years without any tumor recurrence.

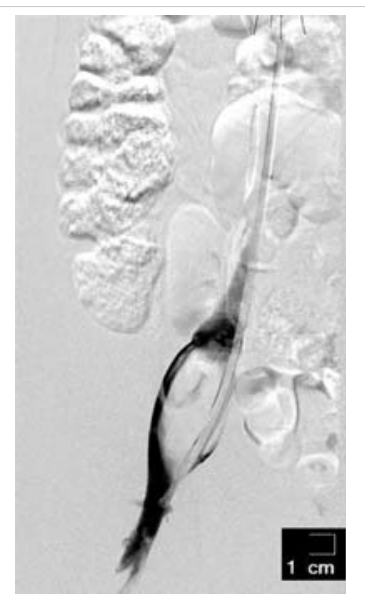

Figure 1: Ultrasound showed right iliac vein, superficial femoral vein, deep femoral vein and popliteal vein.

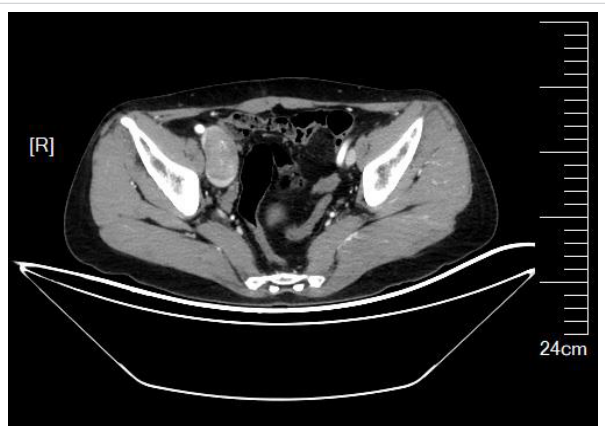

Figure 2: DSA showed a bulging lesion within the iliac vein.

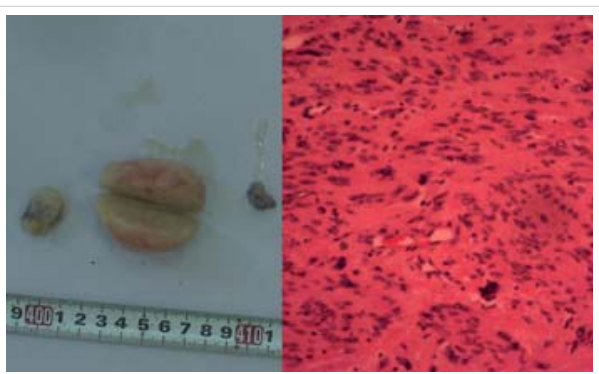

Figure 3: The lesion enhanced heterogeneously during arterial phase.
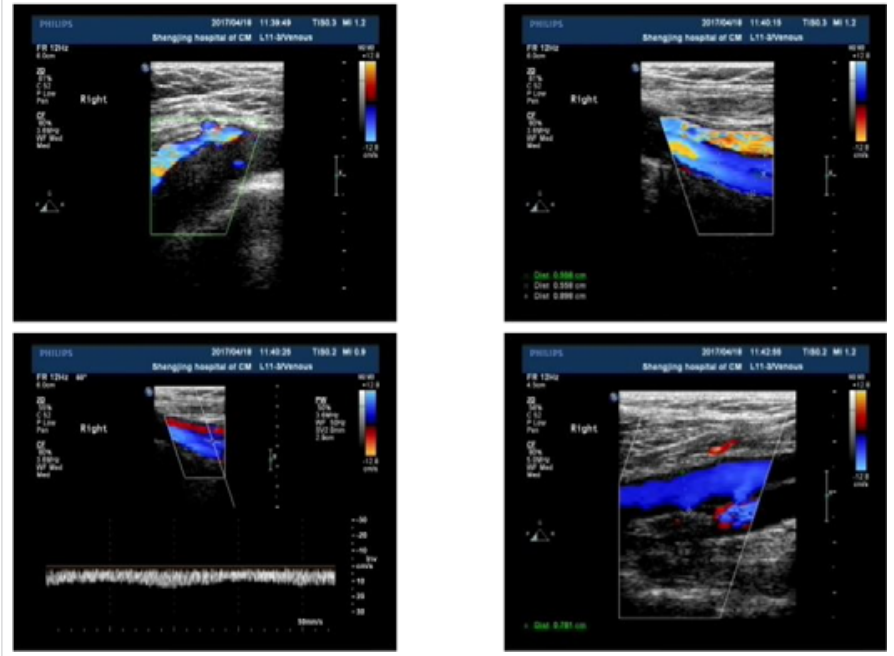

Figure 4: Surgical removed lesions and their microscopic appearance.

\section{Discussion and conclusions}

In retrospect, there might have been some flaws during treatment. First and foremost, was the patient a candidate for catheter-directed thrombolysis? There might have been some problems associated with this patient's Wells' Score. One could argue that the previous DVT might easily be another misdiagnosis of her leiomyoma, and the severity of her calf swelling could also have been disturbed by her left leg atrophy. However, we would still prefer our initial judgment. Her calf swelling was compromised by her previous illness, but it was not entirely an independent feature. The patient had obvious pitting edema at her visit, so one would have good reason to believe that had she been atrophy-free of the other leg, it would still be possible for her right leg to swell more than $3 \mathrm{~cm}$ than her contralateral leg. And we apparently never had dreamed about the possibility of intravascular leiomyoma during her initial visit, so the previous DVT was in and alternative diagnosis was out, and the subsequent ultrasound exam was justifiable.

Deep vein thrombosis (DVT) is a relatively common disease, which can pose a threat to life by causing pulmonary embolism when the clot is large and flowing along the bloodstream. That is why many clinicians tend to treat large thromboses aggressively. Three factors, also known as Virchow's triad, are the major contributors to thrombosis formation, which are blood flow stasis, hypercoagulability and intimal change. Considering the patient's symptoms and travel history, the clinicians were preoccupied with the high probability of DVT, which was supported by the subsequent ultrasound exam. Multiple guidelines suggest that catheterdirected thrombolysis should be strongly considered in acute iliofemoral deep vein thrombus patients with low bleeding risks $[4,5]$. The procedure was a little premature, because a definite imaging had not been acquired at that particular time. Had the CT scan come back, the treatment plan could easily have stepped upon a totally different pathway. 
D-dimers, which are the degradation product of fibrin during fibrinolytic process, are used by clinicians nowadays as a tool to rule out DVT. The diagnostic algorithm for DVT demands the usage of D-dimer testing to exclude thrombus in low probability patient groups. However, for high probability patients, D-dimer testing should yield to diagnostic imaging such as enhanced CT or MRI. The negative predictive value of D-dimer test, or any other tests per se, depends on the prevalence. Thus a normal D-dimer value should not preclude a diagnosis of DVT to be made for high risk patients, though the low value here should have reminded us to further evaluate the validity of the diagnosis.

Inferior vena cava filter is a well-established procedure that is gaining popularity all around the world, despite the fact that in many cases it may be beyond its recommended indications. Many guidelines $[4,6,7]$ endorse its usage in certain situations, such as patients contraindicated to anticoagulation, or recurrent embolism in spite of adequate anticoagulation. However, it is very controversial to apply inferior vena cava filter in other clinical situations, because of the high efficiency of anticoagulation therapy and the potential side-effect caused by filters [8]. But with a person's life at stake, we still concur with the idea to perform such procedure in selected patients, disregarding suggestions based on limited clinical evidences available until more randomized clinical trials bring in their verdict.

There are two hypotheses concerning the pathophysiology of leiomyoma $[9,10]$. One theory suggests that the neoplasm arises from a vessel wall. The other theory implies that they are leiomyomatous cells from the uterine myometrium. Through the wide use of immunohistochemistry, the origin of this invasive benign tumor tends to be attributable to uterine tissue. The majority of patients had a history of uterus leiomyoma. Since the imaging characters supporting leiomyoma are intratumor blood flow signals, the best way to differentiate them, in our opinion, is to look for blood supply within the obstruction dwelling inside the blood vessel, and surrounding circumstances.

To differentiate intravascular leiomyoma from deep vein thrombus based on tradition ultrasound technique is very difficult, adding that the incidence of intravascular leiomyoma is extremely low. But we would advise against using costly and invasive procedures until after a thorough imaging exam to eliminate other possibilities, to avoid the embarrassment we encountered. The oral medication should be sufficient as the initial treatment.

The treatment of intravascular leiomyoma is surgical removal of the entire tumor. If there were tumor residue, there would be a high likelihood of recurrence [2]. Regular follow-up is also highly recommended.

In Conclusion, to differentiate intravascular leiomyoma from deep vein thrombus based on tradition ultrasound technique is very difficult, adding that the incidence of intravascular leiomyoma is extremely low. But we would advise against using costly and invasive procedures until after a thorough imaging exam to eliminate other possibilities, to avoid the embarrassment we encountered. The oral medication should be sufficient as the initial treatment.

\section{Acknowledgements}

The paper has been reviewed and approved by Ethics Committee of Shengjing Hospital of China Medical University.

The authors certify that there is no funding or conflict of interest involved.

\section{Author contributions}

YZ reviewed relevant publications and wrote the manuscript;

FW, who treated the patient as her physician, contributed to the conception of the study;

ZL (Liu), who also treated the patient as her physician, contributed to manuscript preparation;

ZL (Lu), who was department director, contributed significantly with constructive discussions.

\section{References}

1. Xu ZF, Yong F, Chen YY, Pan AZ. Uterine intravenous leiomyomatosis with cardiac extension: Imaging characteristics and literature review. World J Clin Oncol. 2013; 4: 25-28.

PubMed: https://pubmed.ncbi.nlm.nih.gov/23539071/

2. Li B, Chen X, Chu YD, Li RY, Li WD, et al. Intracardiac leiomyomatosis: a comprehensive analysis of 194 cases. Interact Cardiovasc Thorac Surg. 2013; 17: 132-138.

PubMed: https://pubmed.ncbi.nlm.nih.gov/23563052/

3. Cruz I, Joao I, Stuart B, lala M, Bento L, et al. Intravenous leiomyomatosis: a rare cause of intracardiac mass. Rev Port Cardiol. 2014; 33: 735 e731-735.

PubMed: https://pubmed.ncbi.nlm.nih.gov/25455948/

4. Kearon C, Akl EA, Ornelas J, Blaivas A, Jimenez D, et al. Antithrombotic Therapy for VTE Disease: CHEST Guideline and Expert Panel Report. Chest. 2016; 149: 315-352.

PubMed: https://pubmed.ncbi.nlm.nih.gov/26867832/

5. Vedantham S, Sista AK, Klein SJ, Nayak L, Razavi MK, et al. Quality improvement guidelines for the treatment of lower-extremity deep vein thrombosis with use of endovascular thrombus removal. J Vasc Interv Radiol. 2014; 25: 1317-1325.

PubMed: https://pubmed.ncbi.nlm.nih.gov/25000825/

6. Caplin DM, Nikolic B, Kalva SP, Ganguli S, Saad WE, et al. Quality improvement guidelines for the performance of inferior vena cava filter placement for the prevention of pulmonary embolism. J Vasc Interv Radiol. 2011; 22: 1499-1506.

PubMed: https://pubmed.ncbi.nlm.nih.gov/21890380/

7. Jaff MR, McMurtry MS, Archer SL, Cushman M, Goldenberg N, et al. Management of massive and submassive pulmonary embolism, iliofemoral deep vein thrombosis, and chronic thromboembolic pulmonary hypertension: a scientific statement from the American Heart Association. Circulation. 2011; 123: 1788-1830. PubMed: https://pubmed.ncbi.nlm.nih.gov/21422387/ 
8. Malhotra A, Kishore S, Trost D, Madoff DC, Winokur RS. Inferior Vena Cava Filters and Prevention of Recurrent Pulmonary Embolism. Semin Intervent Radiol. 2018; 35: 105-107.

PubMed: https://pubmed.ncbi.nlm.nih.gov/29872245/

9. Norris HJ, Parmley T. Mesenchymal tumors of the uterus. V. Intravenous leiomyomatosis. A clinical and pathologic study of 14 cases. Cancer. 1975; 36: 2164-2178.

PubMed: https://pubmed.ncbi.nlm.nih.gov/1203870/

10. Tierney WM, Ehrlich CE, Bailey JC, King RD, Roth LM, et al. Intravenous leiomyomatosis of the uterus with extension into the heart. Am J Med. 1980; 69: 471-475.

PubMed: https://pubmed.ncbi.nlm.nih.gov/7416191/ 